\title{
"EMBARAZO ABDOMINAL CON FETO VIVO A TERMINO"
}

\author{
Dr. Eduardo Acosta Bendek*
}

La publicación tardía del presente caso ha sido deliberadamente aplazada con el objeto de haber continuado en seguimiento de la niña; hasta el presente en su desarrollo psicofísico no ha presentado ninguna alteración.

El embarazo ectópico avanzado ccupa un lugar muy especial en la patología obstétrica por lo poco frecuente y por las complicaciones graves que registra, lo que conlleva a una alta morbimortalidad fetomaterna. La incidencia en la literatura médica consultaad es de 1: 2075 hasta 1: 20.000 partos $(4,5,7,2,13,10$, 7, 8, 9). El embarazo abdominal puede ocurrir por una implantación primaria del huevo en la cavidad peritoneal, caso muy raro, o secundaria a un aborto tubárico, 0 a una ruptura tubárica o uterina $(13,8)$. En el presente caso de acuerdo a los hallazgos encontrados lo hemos catalogado como un caso de embarazo abdominal secundario a un aborto tubárico.

Nuestro interés en registrar el presente caso además de servir como dato bibliográfico, es el de haber tenido la oportunidad de hacer un seguimiento a largo plazo de la madre y la niña, como también por la conducta seguida en el manejo de la placenta.

\section{HISTORIA CLINICA}

Paciente de 33 años, natural de Barranqui1la. con antecedentes de esterilidad primaria, vida marital desde los 26 años. Menarquia a los 13 años, ciclos regulares: $28 \times 4$; última regla el 30 de Octubre de 1964. Grávida 1, para 0 . Refiere en el presente embarazo haber presentado dolor agudo abdominopélvico tipo cólico asociado a lipotimias, vómitos y distensión abdominal el 15 de Diciembre del mismo año, cuadro éste que cedió con tratamiento médico a las 72 horas de iniciado. El 31 de Diciembre la crisis fue más fuerte con duración de 6 días, recuperándose después de este tiempo. Desde el 20 de Enero de 1965 comenzaron a repetirse las crisis de dolor, mareos y lipotimias en forma más frecuente hasta el 20 de Julio de 1965 en que comenzó a registrar falso trabajo de parto. Fue remitida a mi consulta privada por primera vez el día 31 de Julio encontrando una paciente en mal estado general, anémica y con fascies dolorosas; T.A.: $90 \times 60$, pulso: $110 \times \mathrm{min}$. Al examen obstétrico se encontró abdomen distendido y doloroso lo que dificultó determinar altura uterina $y$ tipo de presentación fetal. Los ruidos cardíacos fetales se percibieron muy velados con una frecuencia de $160 \times$ min. y arrítmicos. Al tacto vaginal se encontró una pelvis clínicamente estrecha, cervix alta, no borrado, de consistencia blanda. Se hizo diagnóstico de impresión de inminencia de ruptura uterina o de embarazo abdominal. Se ordenó su hospitalización inme. diata. El hematocrito de urgencia reveló 27 y la hemoglobina de 7 grs. Se inirió transfusión de $500 \mathrm{cc}$. de sangre y se pasó a cirugía. Se hizo incisión mediana infraumbilcal y se abrió pared por planos según técnica habitual. Se encontró pared abdominal edematosa. Al abrir cavidad peritoneal se observó un útero blando y aumentado de tamaño como para 16 semanas de gestación y que formaba parte de la pared anteroinferior del saco ovular. El saco ovular estaba adherido a epiplón y parcialmente roto; el líquido amniótico era de color ama-

* Decano de División de Ciencias de la Salud de la Universidad Metropolitana. Barranquilla, Colombia S. A. 
rillo verdoso. Dentro del saco ovular se encontró un feto en presentación pélvica y con cabeza en el flanco izquierdo; se extrajo una niña de 2.700 grs. talla 45 cms. y Apgar 7/10; presentó una depresión en la región parieto-occipital derecha y una ligera dolicocefalia como pude verse en la Figura No 1.

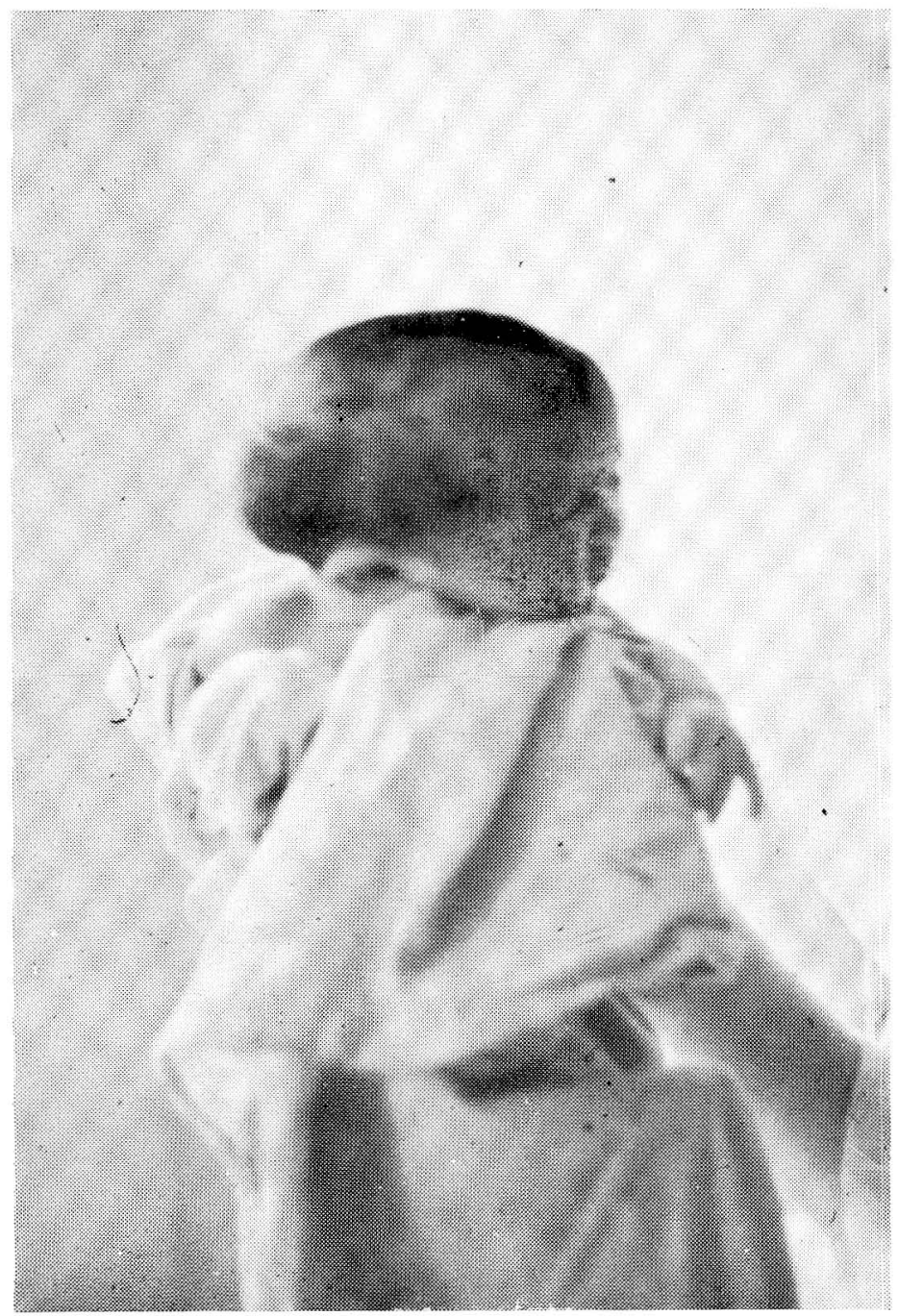

FIGURA $N^{0} 1$ - Niña al nacer.

La placenta estaba localizada en la pelvis so. bre el fondo de saco de Douglas, recto, cara posterior de útero, hoja posterior de ligamento ancho y anexo derecho como se ve en la Figura No 2.
Se hizo debridamiento parcial del saco am. niótico; de la placenta solamente se extirpó la parte desprendida correspondiente al anexo derecho; se hizo hemostasis con sutura continua, el resto de la placenta se dejó en su sitio. 


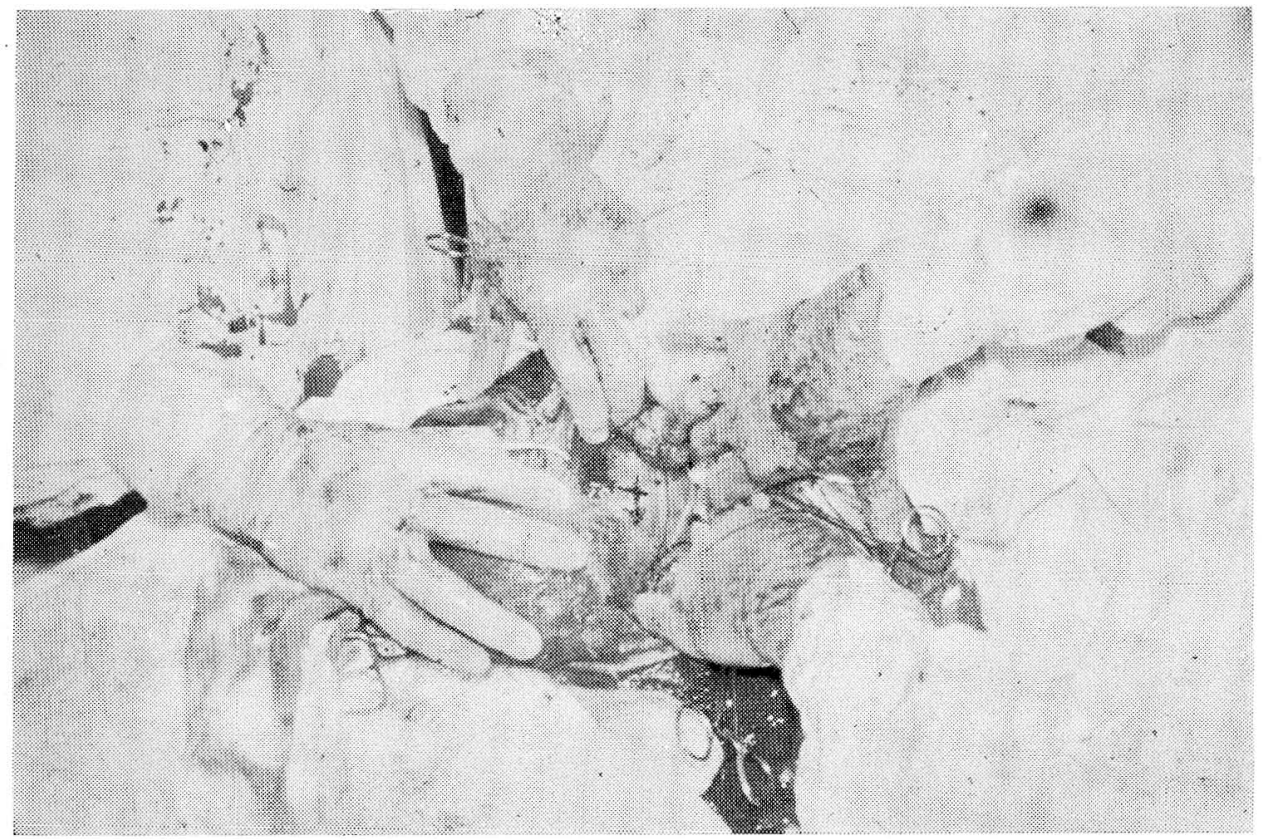

FIGURA No 2

Se dejó un dren de Penrose en fosa ilíaca derecha por la dificultad de hacerlo en el fondo de saco de Douglas por el obstáculo presentado por la pacenta. Se cerró la pared abdominal en 4 planos. Durante el acto quirúrgico se aplicaron $1.500 \mathrm{cc}$. más de sangre. Transoperatorio normal.

El postoperatorio evolucionó más o menos dentro de lo normal, observándose siempre una pequeña distensión abdominal. El día 10 de Agosto se retiró el dren y se dio de alta a la paciente. El 16 de Agosto hizo fiebre y escalofríos y presentó una fístula cecal a través del orificio del dren por lo cual reingresó a la clínica al día siguiente. Se hizo colpotomía y se dejó un dren por 10 días que sirvió para drenar una secreción serosanguinolenta. La fístula se trató médicamente y cerró espontáneamente dos meses más tarde. Mediante exámenes pélvicos mensuales se pudo comprobar la reabsorción total de la placenta a los 8 meses de operada. La menstruación se inició en Diciembre de 1965; de ahí en adelante la paciente se recuperó normalmente.

Consulta nuevamente el 18 de Noviembre de 1969 por amenorrea desde el 15 de Septiembre de 1969, dolor pélvico y vómitos que comenzaron dos días antes. El examen pélvico reveló un útero gestante correspondiente a su amenorrea y sangrado escaso por vagina. El 16 de Diciembre de 1969 es hospitalizada de emergencia por presentar un cuadro de hemorragia intraperitoneal aguda. Durante el acto quirúrgico se comprobó un embarazo ectópico roto de anexo izq. Se observó ciego adherido a peritoneo parietal en la región correspondiente a la fístula anterior. El útero se encontró aumentado de tamaño y miomatoso. Actualmente la señora goza de buenas condiciones de salud y la niña como puede verse en la fotografía № 1 tien 11 años. Los test sicológicos y somáticos no revelan ninguna anormalidad. La deformación de la cabeza casi no se observa.

\section{Comentarios}

El diagnóstico del embarazo ectópico avanzado se dificulta por la sintomatología polifacética que presenta; sin embargo, hay ciertos síntomas y signos en los cuales quiero hacer énfasis y que han sido comunes en 


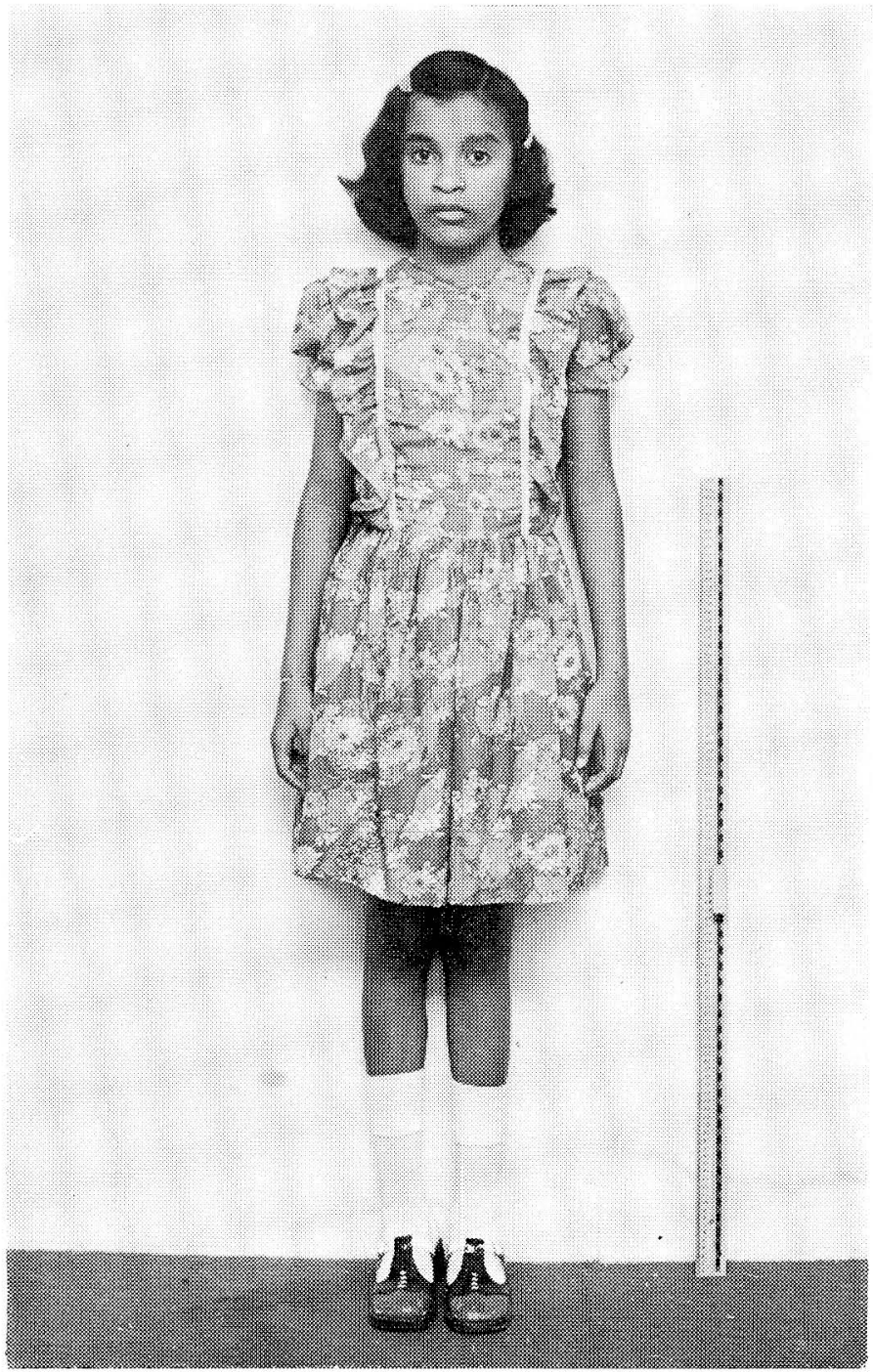

FOTOGRAFIA $N^{0} 1$ - Niña a los 11 años

los casos registrados por mí (1) como son el dolor tipo cólico abdominopélvico asociado a lipotimias, náuseas, vómitos y distensión abdominal que desaparecen a los $3 \circ 4$ días para regresar a las 406 semanas, síntomas que pueden reproducirse durante el tiempo de la gestación du- rante unas 4 o 5 veces. Otro síntoma significativo en la historia de estas pacientes es la infertilidad primaria o secundaria.

El examen pélvico en embarazo avanzado nos dá más información con feto muerto que con feto vivo, ya 
que la delimitación del útero y la localización del feto se hace más fácil con el feto muerto.

Entre los métodos de laboratorio, el de más valor es el de los Rayos X bien sea por medio de la radiografía simple de abdomen en posiciones $\mathrm{AP}$ y $\mathrm{AL}$, complementadas con la histerosalpingografía, nos dá signos patognomónicos de un embarazo abdominal o usando los métodos radiológicos más sofisticados como son: flebografía uterina o la aortografía percutánea transfemoral $(5,3,6)$.

El riesgo materno más serio se presenta de acuerdo a la conducta que se tome con la placenta. De acuerdo a nuestra escasa experiencia y a la cbtenida por otros autores $(4,5,8$, $3)$, la conducta recomendada es la siguiente: si el feto está vivo debe dejarse la placenta en su sitio, haciendo el corte del cordón umbilical a ras de la cara fetal placentaria; si se produce un desprendimiento parcial de la placenta, como sucedió en el caso nuestro, debe hacerse la extirpación de la zona desprendida seguida de hemostasia, cerrar la pared abdominal y no dejar dren. Si el feto está muerto debe tratar de extirparse la placenta siempre y cuando su sitio de implantación y la facilidad de la hemostasia lo permitan, ya que es más fácil y menos riesgoso hacer la extracción de la placenta con feto muerto.

Con relación al tratamiento quirúrgico: si el feto está cerca de la viabilidad se puede esperar bajo estricta vigilancia de la paciente para poder obtener un producto que pueda sobrevivir. Cuando el feto está muerto, si las condiciones de la paciente lo permiten es aconsejable intervenir a las $3 \circ 4$ semanas de muerto el feto.
Con relación a la mortalidad materna, es menor si se deja la placenta in-situ, pero aumenta la morbilidad. Con relación al feto, la mortalidad es alta debido en su mayor parte a que el diagnóstico se hace cuando el feto está muerto; igualmente, la morbilidad es alta debido a las condiciones desfavorables en que se desarrolla el feto.

\section{BIBLIOGRAFIA}

1 ACOSTA BENDEK E.: Comentarios acerca de dos casos de embarazo ectópico a término. Rev. Col. Obst. y Ginec. 8: 195, 1957.

2 ALPIZAR S. y cols.: Embarazo ectópico avanzado. Ginec. Obstet. Mex. 19: 279 1964.

3 BEACHMAN W. y cols.: Abdominal pregnancy at Chanty Hospital in New Orleans. Am. J. Obst. \& Gynec. 84: 1257, 1962.

4 BOTERO URIBE J.: Embarazo ectópico avanzado. Rev. Col. Obst. y Ginec. 11: 307, 1960.

5 CLARK J. y cols.: Abdominal Pregnancy. Am. J. Obst. \& Gynec. 96: 511, 1966.

6 DILAS P. V.: Pelvic angiography in the diagnosis of abdominal pregnancy. Am. J. Obst. \& Gynec. 96: 588, 1966.

7 HUGHES L.: Abdominal Pregnancy associated with intrauterine contraceptive device. Am. J. Obst. \& Gynec. 106, 937, 1970.

8 HRESHCHYSHYN M. Y cols.: Wath is the actual presente-day management of the placenta in late abdominal pregnancy. Am. J. Obst. \& Gynec. 84: 1257, 1962.

9 LAURILA S. y cols.: Un caso de embarazo ectópico a término con niño vivo. Tribuna Médica. 35: 101, 1969.

10 MARTINI A.: Early primary abdominal (peritoneal) pregnancy. Am. J. Obst. \& Gynec. $73: 1139,1957$

11 NAVAS ANGEL G.: Casos curiosos de embarazos ectópicos avanzados. Rev. Col. Obst. y Ginec. 1: 196, 1650.

12 RAMIREZ MERCHAN R.: Embarazo ectópico a término. Rev. Med. y Cirugía. 1946.

13 STUDDIFORD W.: Primary abdominal pregnancy. Am. J. Obst. \& Gynec. 44: 487 1642. 